Modern Physics Letters A

(c) World Scientific Publishing Company

\title{
KAON PHOTOPRODUCTION ON THE NUCLEON WITH CONSTRAINED PARAMETERS
}

\author{
R. NELSON and T. MART \\ Departemen Fisika, FMIPA, Universitas Indonesia, Depok, 16424, Indonesia \\ tmart@fisika.ui.ac.id
}

\begin{abstract}
The new experimental data of kaon photoproduction on the nucleon $\gamma p \rightarrow K^{+} \Lambda$ have been analyzed by means of a multipoles model. Different from the previous models, in this analysis the resonance decay widths are constrained to the values given by the Particle Data Group (PDG). The result indicates that constraining these parameters to the PDG values could dramatically change the conclusion of the important resonances in this reaction found in the previous studies.
\end{abstract}

Keywords: Kaon; photoproduction; coupling constants.

PACS Nos.: 13.60.Le, 25.20.Lj, 14.20.Gk

One of the most intensively studied topics in the realm of hadronic physics is kaon photoproduction. In the last decades a large number of attempts have been devoted to model this reaction. Since this process is not dominated by any single resonant state, the main difference among these models is chiefly in the use of nucleon, hyperon, and kaon resonances. Recently, a large number of experimental data with good quality have been provided by the SAPHIR $\stackrel{1}{1}$, CLAS $^{2}$, LEPS $^{3}$, and GRAAL $\underline{4}$ collaborations. However, the lack of mutual consistency between the SAPHIR and other data found by the recent phenomenological studies has increased the difficulties of the extraction of the "missing resonance" properties. In our previous work we investigated the physics consequence of using each data set $\underline{5}$. It was found that the use of SAPHIR and CLAS data, individually or simultaneously, leads to quite different resonance parameters which, therefore, could lead to different conclusions on "missing resonances". In this paper we extend this investigation by constraining the resonance decay widths to the values given by the Particle Data Group $\underline{6}$. This is intended to approximately account for unitarity corrections at tree-level, i.e., constraining the model by including some information from the leading $\pi$ and $\eta$ channels.

The background amplitudes of the model are constructed from a series of treelevel Feynman diagrams, consisting of the standard $s^{-}, u^{-}$, and $t$-channel Born terms along with the $K^{*}(892)$ and $K_{1}(1270) t$-channel vector mesons. The resonant electric $\left(E_{\ell \pm}\right)$, magnetic $\left(M_{\ell \pm}\right)$, and scalar $\left(S_{\ell \pm}\right)$ multipoles for a state with the mass $M_{R}$, 




Fig. 1. Comparison between angular distribution of differential cross sections obtained from the two fits with CLAS (open circles) SAPHIR (solid squares) and LEPS (solid circles) data.

width $\Gamma_{R}$ are assumed to have the Breit-Wigner form 78

$$
A_{\ell \pm}^{R}\left(W, Q^{2}\right)=\bar{A}_{\ell \pm}^{R}\left(Q^{2}\right) c_{K Y} \frac{f_{\gamma R}(W) \Gamma_{\mathrm{tot}}(W) M_{R} f_{K R}(W)}{M_{R}^{2}-W^{2}-i M_{R} \Gamma_{\mathrm{tot}}(W)},
$$

where $W$ represents the total c.m. energy, $\ell$ indicates the kaon angular momentum, and $\ell \pm \equiv \ell \pm 1 / 2=j$ shows the total angular momentum. The isospin factor $c_{K Y}$ is $\sqrt{3 / 2}$ and $-1 / \sqrt{3}$ for the isospin $3 / 2$ and isospin $1 / 2$, respectively, The factor $f_{K R}$ is the usual Breit-Wigner factor describing the decay of a resonance $R$ with a total width $\Gamma_{\text {tot }}(W)$ and physical mass $M_{R}$, whereas $f_{\gamma R}$ indicates the $\gamma N R$ vertex.

The results of fitting to the SAPHIR or CLAS data, compared with these data, are displayed in Fig. 1 Obviously, the model that fits to the SAPHIR data cannot perfectly explain the CLAS data, and vice versa. It also appears from this figure that the two data sets show the largest discrepancy at $W \approx 1.9 \mathrm{GeV}$ in the forward and backward directions. Consequently, at this energy the total cross section data show a bump (see the upper panels of Fig. 21), which corresponds to the "missing

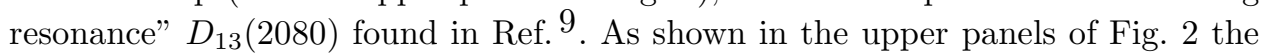
CLAS data show a relatively larger bump compared to the SAPHIR data. Clearly, this implies that the extracted information on the responsible resonances for this peak could be different if we used different data sets.

Contributions of the background terms and the most important resonances are also shown in Fig. 2. Near the production threshold the two well established resonances $S_{11}(1650)$ and $P_{13}(1720)$ show important roles in both fits. This finding emphasizes the results of our previous studies $\underline{510}$. Reference ${ }^{11}$ has also arrived at the same result, except for the $P_{13}(1720)$. Although not too significant, Ref. 11 found that this state is still required in the case of SAPHIR data. Comparison between the two dash-dotted curves in the lower panels of Fig. 22 also confirms that the role of this state is more substantial in the case of SAPHIR data.

Another important state is the $P_{13}(1900)$, a two-star resonance with the total width $\Gamma \approx 500 \mathrm{MeV}$. Although we found that the extracted mass is much larger than $1900 \mathrm{MeV}$, its significant role found in all recent studies $5[10 \mid 11$ is also observed 


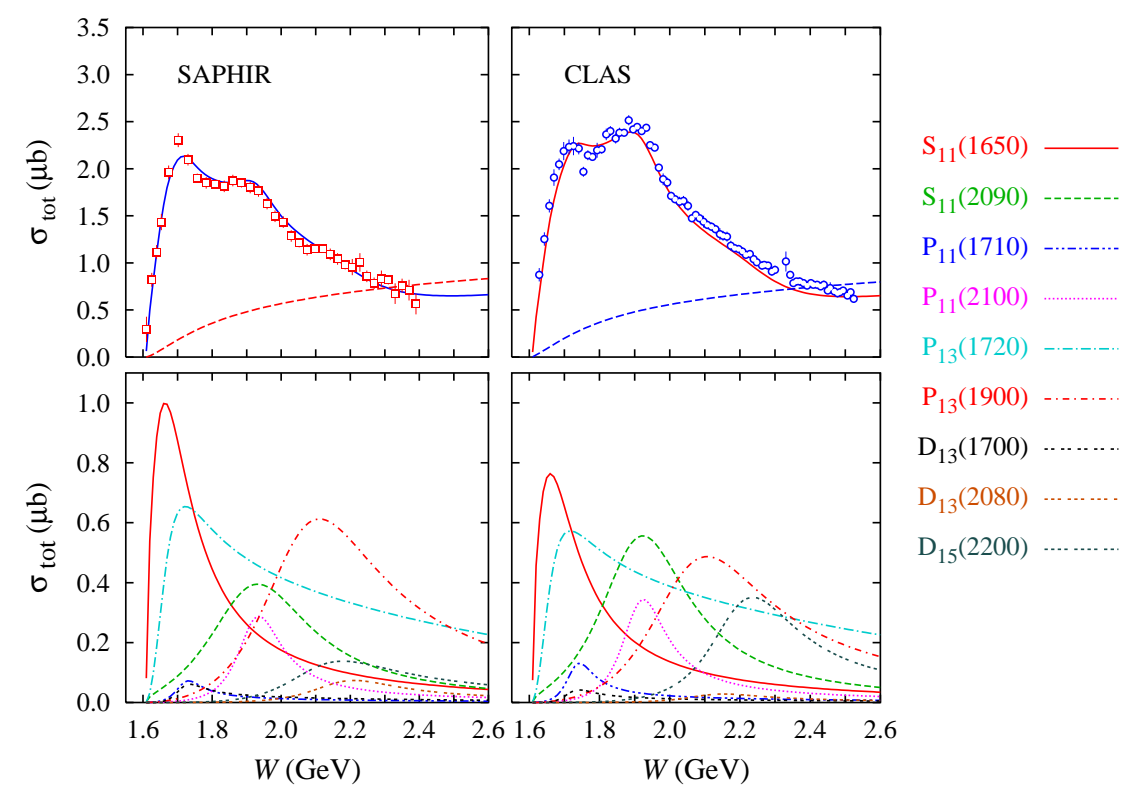

Fig. 2. (Upper panels) Comparison between the calculated total cross sections (solid curves), contributions from background terms (dashed curves), and experimental data. (Lower panels) Contributions from important resonances to the total cross sections.

in the present analysis. On the other hand, the resonance with the same quantum numbers but lower mass $P_{11}(1710)$ plays insignificant role in this process, which is consistent with the finding of our previous work $\underline{5}$.

Compared to the previous studies, the only different result exhibited in Fig.[2] is the origin of the second peak in the total cross section. As clearly shown, this peak originates from the contributions of the $S_{11}(2090)$ and $P_{11}(2100)$ states. Most of the recent investigations found that this peak indicates a "missing" $D_{13}$ resonance with a total width $\Gamma$ varies from 165 to $570 \mathrm{MeV}$. Clearly the effect of constraining the fitted parameters is quite significant in this case. Therefore, it is quite important to address this issue in the future single channel analyses of kaon photoproduction.

It has been found that the inclusion of the new CLAS $C_{x}$ and $C_{z}$ data reveals the role of the $S_{11}(1650), P_{11}(1710), P_{13}(1720)$, and $P_{13}(1900)$ resonances for the description of these data 10 . In this study we also investigate the effects of these data on our model. The importance of the individual resonance for the fits with and without these data is represented by $\Delta \chi^{2}=\left|\chi_{\text {All }}^{2}-\chi_{\text {All }-N^{*}}^{2}\right| / \chi_{\text {All }}^{2} \times 100 \%$ in Fig. [3, where $\chi_{\mathrm{All}}^{2}$ is the $\chi^{2}$ obtained by using all resonances and $\chi_{\mathrm{All}-N^{*}}^{2}$ is the $\chi^{2}$ obtained by using all but a specific resonance. Obviously, constraining the free parameters in the fits changes the conclusion of the previous analyses. Only the $P_{13}(1720)$ seems to be still important in both cases, whereas the $P_{13}(1900)$ is only important in the fit without $C_{x}$ and $C_{z}$ data. The near-threshold resonance $S_{11}(1650)$ is found to be 

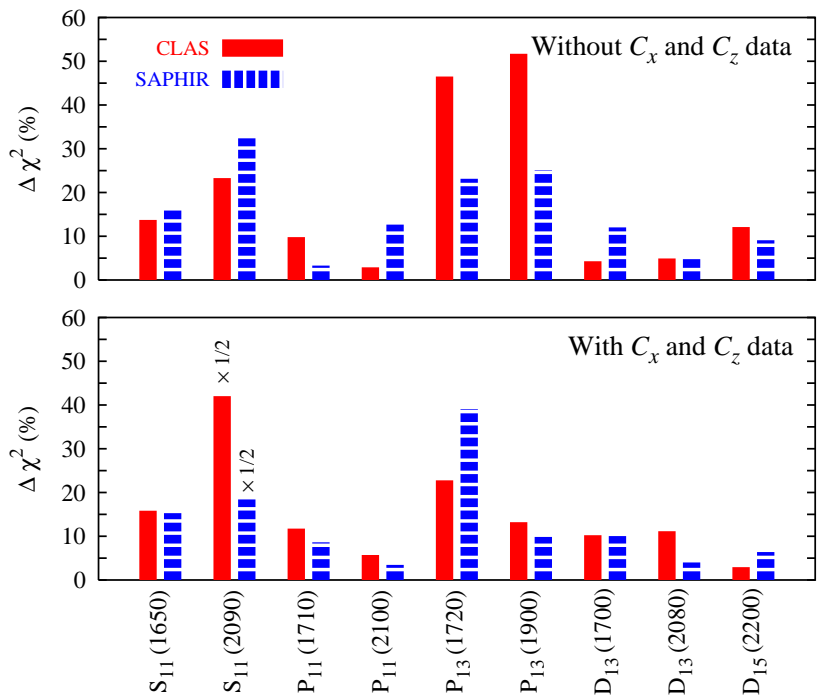

Fig. 3. (Upper panel) The significance of individual resonances for fitting to the CLAS and SAPHIR data. (Lower panel) As in the upper panel, but including the CLAS $C_{x}$ and $C_{z}$ data.

relatively important in both cases. We also note that in this study the $S_{11}(2090)$ is found to be quite important in both cases. The importance of this state has been actually found by the former study $\left[5\right.$, although with a smaller $\Delta \chi^{2}(\approx 6 \%)$.

In conclusion we would like to say that the use of different data sets could lead to different conclusions on the important resonances required in the kaon photoproduction. Furthermore, constraining the free parameters in the multipoles model for this process results in a significant effect and could dramatically change the conclusion found in the previous analyses. A more detailed study is currently underway and the result will be published elsewhere in the near future.

The authors acknowledge the support from the University of Indonesia.

\section{References}

1. K.-H. Glander et al., Eur. Phys. J. A 19, 251 (2004).

2. R. Bradford et al., Phys. Rev. C 73, 035202 (2006).

3. M. Sumihama et al., Phys. Rev. C 73, 035214 (2006).

4. A. Lleres et al., Eur. Phys. J. A 31, 79 (2007);

5. T. Mart and A. Sulaksono, Phys. Rev. C 74055203 (2006).

6. C. Amsler et al., Phys. Lett. 667, 1 (2008).

7. D. Drechsel, O. Hanstein, S. S. Kamalov and L. Tiator, Nucl. Phys. A 645, 145 (1999).

8. L. Tiator et al., Eur. Phys. J. A 19, 55 (2004).

9. T. Mart and C. Bennhold, Phys. Rev. C 61, 012201 (2000).

10. T. Mart, Few-Body Syst. 42, 125 (2008).

11. B. Julia-Diaz, B. Saghai, T. S. Lee and F. Tabakin, Phys. Rev. C 73, 055204 (2006). 